(C) [2005] IEEE. Reprinted, with permission, from [David G. DORRELL, Calculation and effects of end-ring impedance in cage induction motors, Magnetics, IEEE Transactions on, Volume: 41 , Issue: 3 March 2005]. This material is posted here with permission of the IEEE. Such ermission of the IEEE does not in any way imply IEEE endorsement of any of the University of Technology, Sydney's products or services. Internal or personal use of this material is permitted. However, permission to reprint/republish this material for advertising or promotional purposes or for creating new collective works for resale or redistribution must be obtained from the IEEE by writing to pubspermissions@ieee.org. By choosing to view this document, you agree to all provisions of the copyright laws protecting it 


\title{
Calculation and Effects of End-ring Impedance in Cage Induction Motors
}

\author{
David G. DORRELL, Member, IEEE
}

\begin{abstract}
This paper describes a method for calculating the end-ring impedance of a squirrel cage induction motor using the method of images. The method is verified using finite element analysis. A second method is also discussed but was found to be too simple to be accurate. The second part of the paper addresses the effect of this impedance variation on the motor performance with particular attention being paid to the starting torque.
\end{abstract}

Index Terms - end-ring, induction motor, rotor impedance

\section{INTRODUCTION}

$\mathrm{T}$ HIS paper investigates a simple but effective method to calculate the end-ring impedance (essentially the resistance) of a cage rotor in an induction motor. This method can be used in analytical models (a typical per-phase circuit for a 3-phase machine is shown in Fig. 1) or 2d finite element models where a lumped equivalent circuit parameter for the end-ring impedance can be put into the circuit. The values of the impedances in the equivalent circuit in Fig. 1 dictate the motor performance. This is similar to a transformer equivalent circuit; the primary consists of the 3-phase stator winding while the secondary consists of the cage structure (aluminum or copper) on the rotor. The rotor cage bars lie in slots or holes just under the rotor surface while the bar ends are connected by rings at each end which are either part of the casting for an aluminum cage or brazed on if fabricated from copper. The rings may be butted up against the rotor core-pack (cast) or be located away from the core-pack to allow brazing to take place. The rotor impedance will therefore consist of the bar resistance and leakage reactance, the end-ring resistance and leakage reactance, the differential reactance and a skew reactance (due to skew of the cage down the axial length). The rotor impedance tends to be dominated by the bar impedance and differential reactance although the end-ring can make a difference to the motor performance. Often the end-ring reactance is ignored and a simple resistance term, based on the segment length and area and also the pole pitch of the segment, is used to calculate the resistance, i.e., it is the effective dc resistance of the end-ring. At rated speed and for the fundamental forward-rotating field, this is usually a good calculation. At start (for a direct on-line start of a grid-

D. G. Dorrell is with the Department of Electronics and Electrical Engineering, The University of Glasgow, Glasgow, G12 8LT, UK. (phone: +44 1413302768; fax: +441413303158; e-mail: d.dorrell@elec.gla.ac.uk). connected machine where the frequency of the supply is grid frequency) there may be substantial skin effect in the ring in a large machine so that an ac resistance should really be used. It must be stated, though, that the amount contributed by the endring resistance and reactance to the rotor impedance will vary from machine to machine; if the machine has end-rings with large cross-sectional areas then the contribution can be relatively small, whereas if high starting torque is required, then one technique is to decrease the end-ring cross section, which increases the rotor resistance and hence starting torque (increasing the rotor resistance will usually increase the starting torque). If using FEA or an analytical package such as the SPEED PC-IMD package (developed by the University of Glasgow, UK) then skin effect in the rotor bar is either implicit in the calculation method (FEA) or can added as required when modeling large machines (SPEED). However it is most unusual to incorporate a skin-effect allowance for the end-ring reactance. It is often found to be the case that when measuring the power, current and power factor of an induction machine when starting, it is possible to obtain two of these components accurately by modeling, but the third is inaccurate by several percentage points. Refining the circuit parameters to a greater degree of accuracy will obviously improve the accuracy of modeling. The author has already investigated one additional refinement of the skew reactance term in small motors [1] by incorporation of inter-bar current into the modeling process of the fundamental forward-rotating component in a balanced 3phase motor (and this will be extended to allow for the effects on the harmonic components in Alger's equivalent circuit [2] and backwards rotating component when unbalanced). This paper, in a similar vein, will address further refinement of the rotor impedance and show the affects of frequency variation on the end-ring impedance.

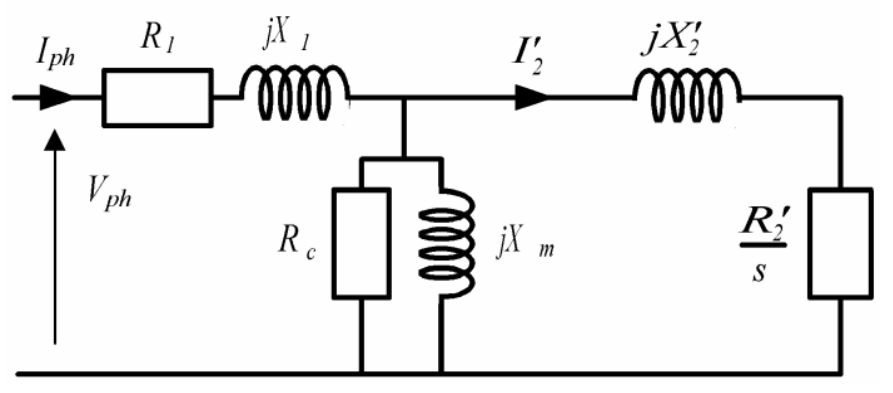

Fig. 1. Per-phase equivalent circuit for a 3-phase induction motor 
The work reported here uses a model first developed by Williamson and Mueller [3] and develops it further to incorporate a non-uniform distribution of end-ring elements. This is because the change in current density is greater at the end-ring edges so that there needs to be a higher density of elements in the edge region. The work compares the results to a finite element calculation and also a more trivial calculation technique using a method by Stoll [4] which is not necessarily a method for calculating end-ring reactance. The Stoll method is shown to be inappropriate.

These methods for modeling the end-ring reactance assume that the current flows in the circumferential direction. While this is not necessarily correct since the point where the bar joins the end-ring will lead to a redistribution of the current, this is a $3 d$ problem of some complexity. This was studied by Yamazaki [5] as part of an overall 3d FEA study of an induction motor. However the aim of this paper is to present a more straightforward model to improve the accuracy of the design. Kim et al [6] also conducted a 3d FEA analysis of the end-ring region and illustrated that the current distribution across the ring is considerably altered and that the resistance is increased. A 2d FEA approach was used by Chan et al [7] (also by Williamson and Mueller as a verification process). While several people have studied the rotor as a complete $3 \mathrm{~d}$ simulation, the problem with this approach is that the mesh region has to be fine in the bar/end-ring join to get accurate current distribution and also there is no inter-bar current in the model. This was illustrated by Kometani et al [8] who carried out a full $3 \mathrm{~d}$ solution on a skewed rotor but still obtained a 5.5 $\%$ error in the phase current. This work also showed the rms current in the end-ring which is not necessarily the correct component to illustrate; while the total rms current in the endring may not change greatly, since it is almost current fed, the impedance will change since the end-ring voltage is floating. The end-ring can be considered as being current fed because its impedance is only one component of a set of seriesconnected impedances in the rotor loop of the equivalent circuit. The bar resistance and leakage inductance, differential leakage reactance and skew reactance also have to be included in the rotor impedance. Therefore even if there is a large change in end-ring impedance, there may still be only a small change in total rotor impedance.

The second part of this paper will assess the effect of ring-ring impedance adjustment due to skin effect on motor performance. This will be done using two large machine models.

\section{Analytical CAlCulations - Elemental Method AND STOLL ANALYSIS}

\section{A. Elemental Analysis Using the Method of Images}

This model is straightforward and uses the Method of Images so that the end-ring, which is close or butted-up to the end of the rotor core-pack, is simulated using a single ring and an image of this ring on the opposite side of the rotor core edge. Fig. 2 shows the filamentary representation of the end-ring with its image ring. The edge of the rotor core represents a symmetrical centre of the model and flux will cross this boundary in the perpendicular direction; since the boundary is assumed to have infinite permeability then the flux distribution (and hence the solution) in the right-hand plane will be the same if we replace the iron boundary with this image ring. This is due to symmetry. The development of the theory will initially use filaments that are equally spaced and equally sized as used in [3]. However, as will be shown later, this is modified to use different sizes of elements as shown in Fig. 2. This is because the current will flow at the edges of the ring if the skin effect is high and the change of current density from element to element is large; whereas in the centre of the ring the current density is more uniform under all conditions so that the density of elements can be less. A matrix inversion is used to solve this problem and even with a modern computer the solution can take a period of time if the element density is high and many solutions are required over a range of operating conditions (such as a full torque/speed curve).

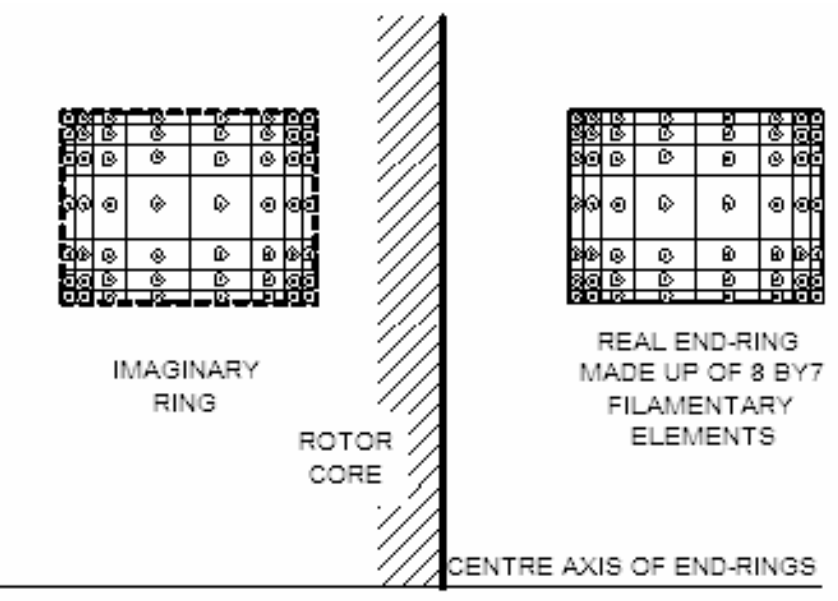

Fig. 2. Filamentary representation of the end-ring and image

In a squirrel-cage rotor the current flows between bars via the two end-rings. The end-ring current is much higher than the bar current so that the cross-sectional area of the end-ring is likewise much higher. At standstill the frequency of the current in the ring is the same as the motor supply frequency. This frequency will decrease as the machine approaches the synchronous speed until zero is reached. Therefore $f_{r}=s f_{s}$ where $s$ is the slip between the rotor speed and the synchronous speed. If the cross-section is large and susceptible to skin effects then the AC resistance and leakage reactance will be a function of the machine speed. There is much literature about calculating the bar resistance and leakage reactance when skin effect is present (where the current travels at the top of the bar and increases the resistance and decreases the leakage reactance). However there is little on end-ring calculation except for [3] and [5] to [8]. Reference [3] is used as the basis of the analytical model here although it is modified.

The end-rings in induction motors come in a variety of shapes but here we will study a simple rectangular shape, which varies in distance from the stator core pack. A short 
study was conducted by Smith [9] into the effect of end-ring shape, although there is probably a need for more in-depth study in this area. In cast-aluminum rotors the end-rings tend to be butted-up to the core-pack whereas with fabricatedcopper cages the bars often protrude so that the end-rings are moved away from the core-pack.

Firstly let us only consider the real ring and then add in the imaginary ring at the end of the analysis. Initially we can assume that the height above the rotor axis of the inner radius is $R a d, H$ is the width of the ring in the radial direction, the width of the ring in the axial direction is $D$. The dimensions are shown in Fig. 3 (although the axes are rotated 90 degrees counter-clockwise compared to Fig. 2). If the ring is split into filamentary elements of uniform size that have coordinates of $\left(h_{m} d_{n}\right)$ then

$$
h_{m}=\operatorname{Rad}+\frac{m H}{M}
$$

and

$$
d_{n}=\frac{n D}{N}
$$

where $M$ is the number of element layers in the radial direction, $N$ is the number of element layers in the axial direction, $m=1$ to $M$ and $n=1$ to $N$. The self and mutual inductances can be calculated and an impedance matrix formed:

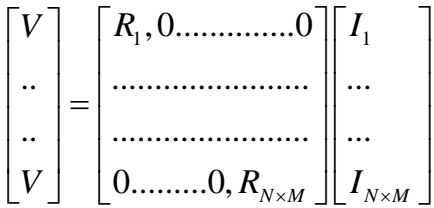

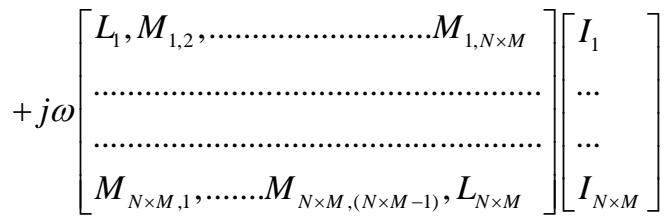

For a set of excitation voltages, the matrix can be solved and a ratio of ac to dc resistance obtained. The resistance of each element is:

$$
R_{n \times m}=\rho \frac{2 \pi h_{m} M N}{H D}
$$

The mutual inductance [3] is

$$
M_{n m, o p}=\mu \sqrt{h_{m} h_{o}}\left[\left(\frac{2}{k}-k\right) K(k)-\frac{2}{k} E(k)\right]
$$

where

$$
k^{2}=\frac{4 h_{m} h_{o}}{((n-p) D / N)^{2}+\left(h_{m}+h_{o}\right)^{2}}
$$

The functions $K(k)$ and $E(k)$ are definite integrals which, when the element sizes are small so that $k$ is nearly unity, approximate to

$$
K(k)=\ln \left(\frac{4}{\sqrt{1-k^{2}}}\right)
$$

and $E(k)=1$. This is obtained from [9] and is different from that quoted by Williamson and Mueller in [3] where they state that $K(k)$ and $E(k)$ are complete elliptical integrals and evaluated using $4^{\text {th }}$ order polynomials as illustrated in [11]. However a more straightforward method uses (7) and $E(k)=1$ which proves to be a satisfactory approximation. From [9], the self inductance of a ring of rectangular section can be approximated to:

$$
L_{n m}=\mu h_{m}\left[\ln \left(\frac{8 h_{m} M}{H}\right)-2\right]
$$

which is again a more straightforward expression from that used in [3].

The total current is then the sum of all the current elements and the ac power is the sum of the power losses in each element so that

$$
I_{\text {total }}=\sum_{n=1}^{N} \sum_{m=1}^{M} I_{m \times n}
$$

and

$$
P_{\text {total }}=\sum_{n=1}^{N} \sum_{m=1}^{M} I_{m \times n}^{2} R_{m \times n}
$$

The ac resistance and reactance can be obtained from

$$
R_{a c}=\frac{P_{\text {total }}}{I_{\text {total }}^{2}}
$$

and

$$
X_{a c}=\frac{Q_{\text {total }}}{I_{\text {total }}^{2}}
$$

where

$$
Q_{\text {total }}=\sqrt{V I_{\text {total }}-P_{\text {total }}}
$$

The analysis so far has only considered the real ring. However, the image ring can now be placed at the other side of the boundary at the same distance from the boundary. The 
filamentary mutual inductances between the real and imaginary rings can be calculated using the same set of equations using the correct geometrical distances between real and imaginary filaments. The set of self and mutual inductances for the imaginary ring itself will be identical to the real ring due to symmetry. Filaments for the imaginary ring can then included in the impedance matrix and the matrix solved to obtain the current values. However, when using this method, the mirrorimage elements in the imaginary ring will have the same currents as the real elements, so that the matrix size will not increase due to the inclusion of the imaginary ring and will remain at $M \times N$ since the current vector defines the real and imaginary ring currents.

This algorithm can easily be implemented in an environment such as MATLAB. This is very simple due to the straightforward geometry and also, since no saturation is included in the model, the impedances are independent of driving voltages and currents. This means that the matrix can be can be solved using an arbitrary voltage set to produce an end-ring impedance that can be placed directly into a perphase equivalent circuit or coupled circuit of a $2 \mathrm{~d}$ finite element solution.

\section{B. Stoll Method [4]}

This method was initially investigated since it appeared to be a straightforward method to implement. However, it does prove to be inaccurate but it is included here for completeness. If the bar is narrow and the radius high then a 1-dimensional model can be implemented. In this case $D \gg H$ and the variation of the current in the radial direction is limited. Therefore we can approximate the current density to:

$$
J=\frac{\alpha I}{2 H} \frac{\cosh \alpha y}{\sinh \alpha \frac{D}{2}}
$$

where

$$
\alpha=\frac{1+j}{\delta}
$$

and the skin depth

$$
\delta=\sqrt{\frac{\rho}{\pi f \mu}}
$$

In this case $y$ is the axial distance, $f$ is the frequency and material is copper. The current $I$ is the total conductor current. From these equations, the resistance ratio is

$$
\frac{R_{a c}}{R_{d c}}=\frac{\gamma}{2} \frac{\sinh \gamma+\sin \gamma}{\cosh \gamma-\cos \gamma}
$$

where $\gamma=D / \delta$

If the solution is two-dimensional then a common approximation is to solve the equations in each direction and then use superposition to obtain a value for the ac-to-dc resistance ratio.

\section{Finite ElEmEnt MethoD}

This method is used to verify the accuracy of the two analytical models. Fig. 3 shows a model of the end-ring with $70 \mathrm{~mm}$ axial width and $10 \mathrm{~mm}$ radial depth. Since the Stoll method does not include the rotor iron then this is omitted in the initial model in Fig. 3. However it is added later to test the filamentary method. The FEA is axi-symmetric around the rotor axis. The inner radius of the ring is $100 \mathrm{~mm}$. These dimensions can be automatically varied in order to generate look-up tables. The reason for choosing a thin ring at this stage is to assess the accuracy of the Stoll 1-dimensional expression for the current distribution. The diagram is rotated by 90 degrees to the left compared to Fig. 2.

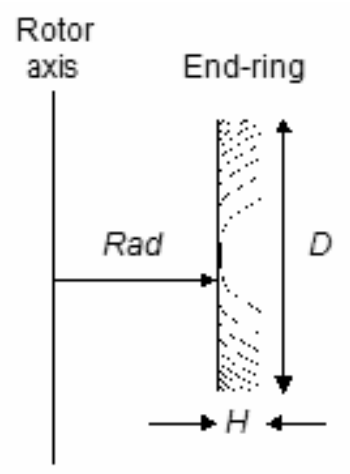

Fig. 3. Ring with dimensions of $70 \mathrm{~mm}$ (axial) by $10 \mathrm{~mm}$ (radial) and 100 $\mathrm{mm}$ inner ring radial. Lines of constant current density at a frequency of 50 $\mathrm{Hz}$.

The ring was excited from an external ac voltage source of 10 $\mathrm{V}$ rms. To obtain realistic current levels and allow for steel saturation of the rotor end-core, the voltage can be varied until the total current in the ring is approximately equal to that in the actual machine. The ac resistance was obtained by calculating the mean loss in the ring and the total rms current in the ring so that

$$
R_{a c}=\frac{P_{\text {mean }}}{\left|I_{r m s}\right|^{2}}
$$

and

$$
R_{d c}=\rho \frac{2 \pi(\operatorname{Rad}+H / 2)}{H D}
$$

which allows for the calculation of the ac-to-dc resistance ratio.

The current density for this ring at $50 \mathrm{~Hz}$ is shown in Fig. 4. It can be seen that there is a ratio of about $2: 1$ between the maximum and minimum current densities at the different radii. The ratio $\mathrm{Rac} / \mathrm{Rdc}$ was 1.09 . 


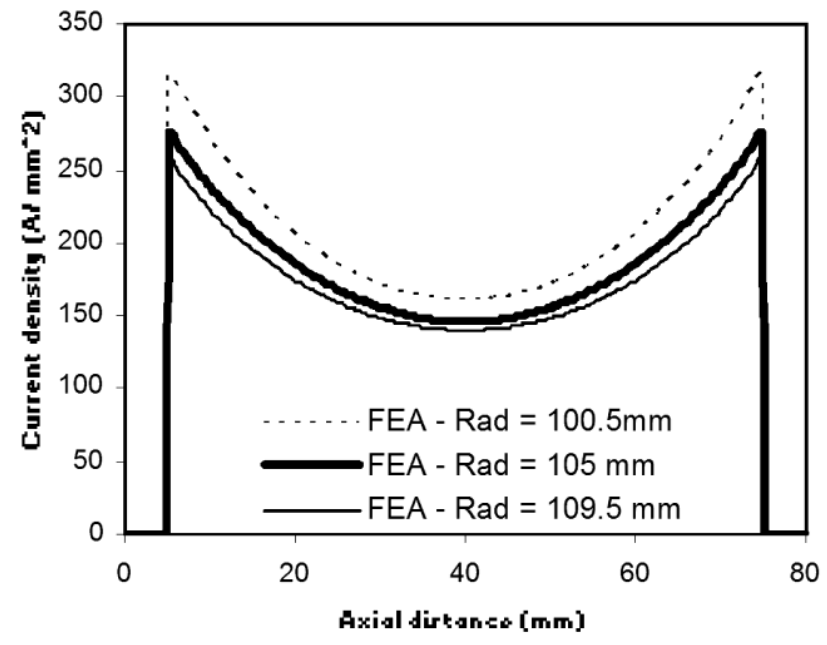

Fig. 4. Current density profile for ring in Fig. 3 using FEA

\section{COMPARISONS OF SIMULATIONS}

\section{A. Comparison of Methods with no Rotor Core and Narrow End-Ring}

Fig. 5 shows a comparison of the three methods for the 10 by $70 \mathrm{~mm}$ ring. It can be seen that the current density of the FEA and filamentary methods compare well with each other but the Stoll method gives poor results. This is reflected in the values of Rac/Rdc which are FEA $=1.09$, Elemental $=1.11$, Stoll $=$ 3.75. The elemental method uses elements of $1 \mathrm{~mm}^{2}$ rather than the graded sizes as shown in Fig. 2. Detailed comparisons between the Stoll and FEA methods showed poor correlation for many of the geometries, which prompted the use of the elemental method. If $1 \mathrm{~mm}^{2}$ elements are used then 700 elements are required. However, by inspection of Fig. 5 it is clear that the centre region does not require this high density of elements whereas the edge regions require a high density. Therefore, to reduce the computation time a method was adopted where the size of the elements was graded and the number of elements assigned as a function of the ring dimensions compared to the skin depth.

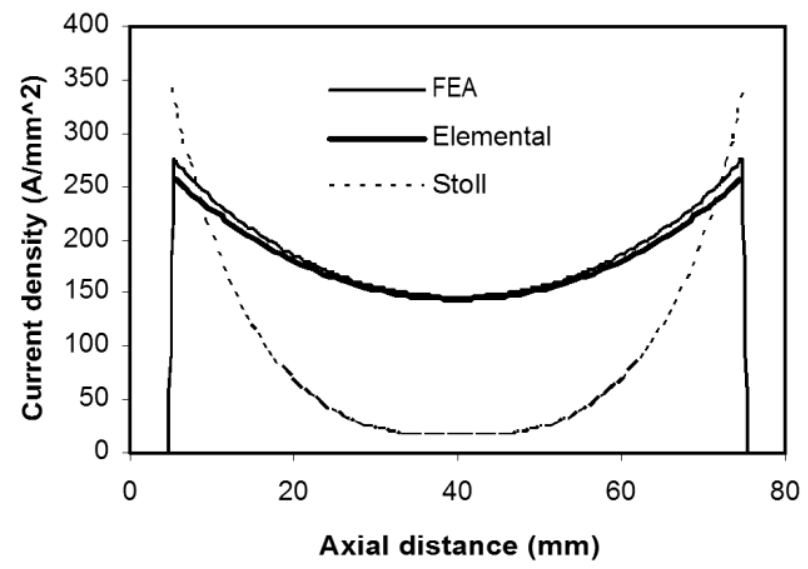

Fig. 5. Current density variation, using three different methods, for the ring in Fig. 3 and a radius of $105 \mathrm{~mm}$.
Experience shows that a maximum of about 15 by 15 elements should suffice but it may be less depending on the skin effect. The solution is then fast if skin effect is negligible.

For the $n^{\text {th }}$ element in the ring width $D$ then the total number of elements $N$ is calculated using the equation

$$
N=\operatorname{int}\left(\frac{K_{N} D}{\delta}\right)
$$

where $K_{N}$ is a refinement integer - the higher the number the more elements are obtained. The reference width of the element is then

$$
w_{n}^{\prime}=\exp \left|\frac{(N / 2-0.5+n) D K_{C}}{\delta N}\right|
$$

where $K_{C}$ is a concentration factor - the higher the factor the more concentrated the elements at the edge. In the simulations here we are using $K_{N}=3$ and $K_{C}=2$. The total reference width is

$$
W_{N}^{\prime}=\sum_{n=1}^{N} w_{n}^{\prime}
$$

so that the actual width of the $n^{\text {th }}$ element is

$$
w_{n}=w_{n}^{\prime} \frac{D}{W_{N}^{\prime}} .
$$

A similar set of equations exists for the radial thickness $H$ to define the $M$ number of radial elements of the ring. Hence we now have a set elements defined across the ring cross section which numbers $N \times M$ that are non uniform in size and concentrated at the edge of the ring.

\section{B. Comparison of Graded Element Method with FEA Including Rotor core}

The model was verified using Cedrat Flux2D. Because of the number of solutions necessary and the continually changing nature of the geometry when comparing end-ring sizes and locations then only one solution was manually obtained. The Flux2D .spi files from this simulation were used as skeleton programs and incorporated into PASCAL programs specially written to automatically generate a set of .spi files for each solution (incorporating the changes in geometry and frequency). The node densities along the edges were made a function of the edge length to produce a good mesh in each case. The whole set of simulations could then be run automatically one after the other using a bat file (again generated automatically by use of a PASCAL program). Therefore several hundred solutions could be run in one operation. The Flux2D geometry editor used was PREFLU. 


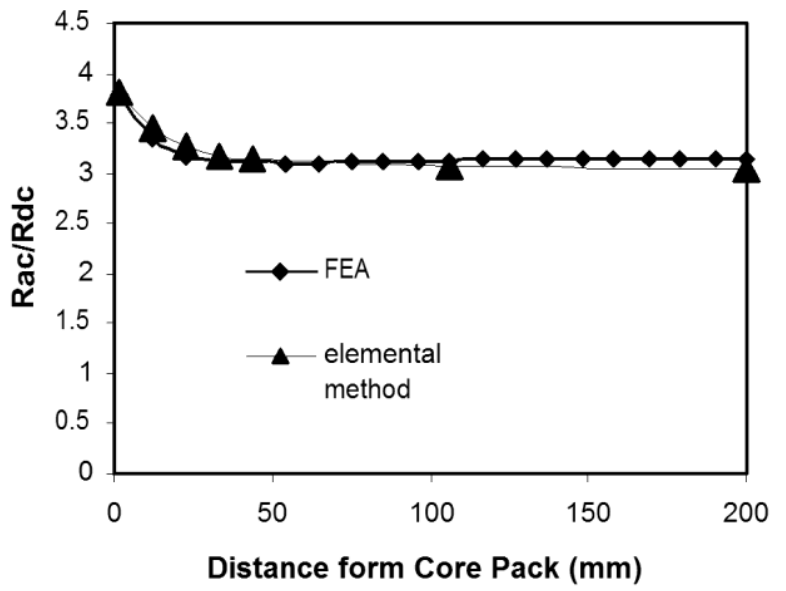

Fig. 6. Comparison of FEA and elemental method. End-ring is $30 \mathrm{~mm}$ square with inner radius of $100 \mathrm{~mm}$ and frequency of $400 \mathrm{~Hz}$

Fig. 6 shows the change in resistance ratio (Rac/Rdc) with distance from the core-pack of the ring. The frequency was increased to a high level $(400 \mathrm{~Hz}$ is airplane power system frequency) to emphasize the effect. Obviously a ring of this size suggests a large machine that would not normally be found on an airplane.

\section{Current Density Across the Ring}

Fig. 7 shows the variation of current diagonally across the bar for the FEA analysis from the inner-radius corner away from the core to the outer-radius corner nearest the core (Fig. 5 illustrated the coherence between the FEA and analytical model). While the densities are high this is purely an arbitrary level. The FEA was a linear solution and a nominal voltage of $10 \mathrm{~V}$ was applied to the ring as a driving voltage. The voltage magnitude does not affect the current distribution in these simulations. The current is highest on the inner surface of the ring and furthest away from the core. If the ring is placed close to the rotor core then the current density is increased due to the increased skin effect. This can be used to shape the ring and produce different impedance characteristics. Fig. 8 shows the current density along the inner edge of the ring. Figs. 7 and 8 show that there is little current in the centre of the ring in these conditions and it rises steeply towards the edges. It should be borne in mind that this simulation uses an extremely high frequency (airplane power system frequency), which are used deliberately order to illustrate the effect and why we wish to concentrate the elements at the edges.

\section{EFFECT ON MACHINE PERFormance}

\section{A. Motor Specifications}

Two 3-phase motor designs were used to investigate the effects of the end-ring skin effect. The first is a $100 \mathrm{hp} \mathrm{4-pole}$ $60 \mathrm{~Hz}$ motor and the second is a $300 \mathrm{hp} \mathrm{12-pole} 60$ motor. Relevant details are given in Table I. These were obtained using the SPEED software from The University of Glasgow which has been found to be accurate in calculating the parameters in machines of this type. This software takes into account deep bar effects in the rotor bars (as can be observed) although it does not include skin effect in the end-rings. It can be seen, by looking at the referred rotor bar resistance at rated load and at standstill (where the current frequency in the rotor changes from slip frequency [ $=$ p.u. slip $\times$ supply frequency] to supply frequency), in conjunction with the referred rotor resistance, that the $100 \mathrm{hp}$ has a high variation of the rotor impedance due to deep bar effects compared to the $300 \mathrm{hp}$ motor.

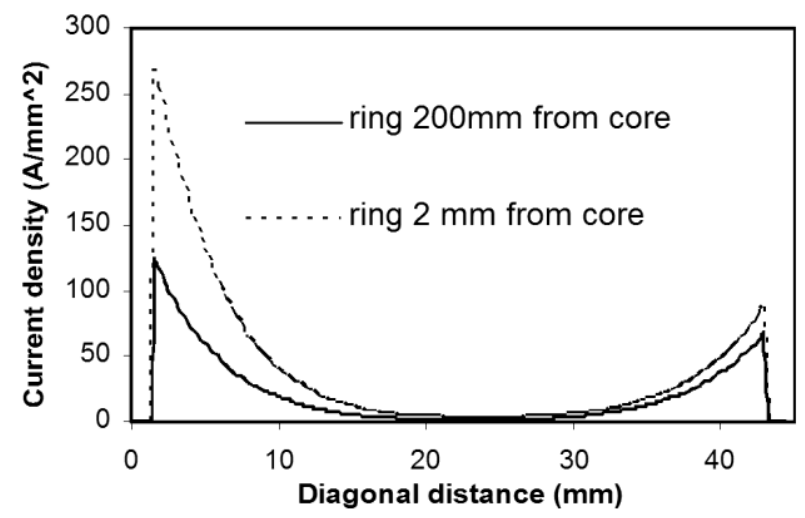

Fig. 7. Current density across diagonal of $30 \mathrm{~mm}$ by $30 \mathrm{~mm}$ ring at $400 \mathrm{~Hz}$. Curves start at inner corner away from the stack.

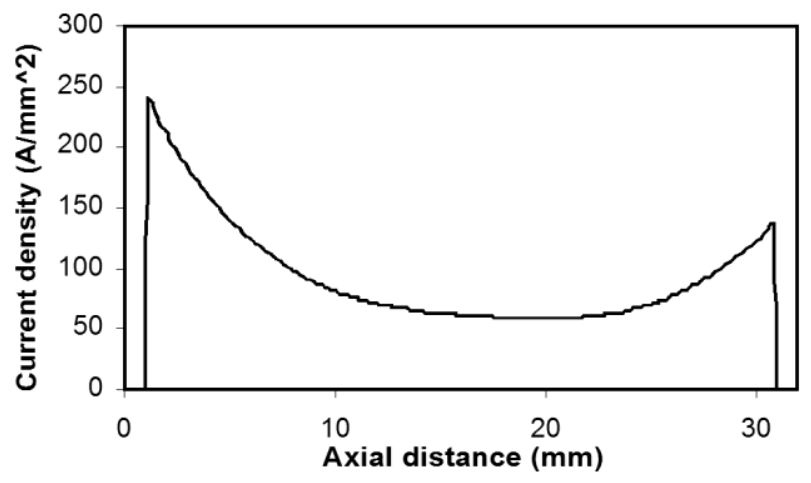

Fig. 8. Current density on inner surface of $30 \mathrm{~mm}$ by $30 \mathrm{~mm}$ ring at 400 Hz. Curves start at corner away from the stack and the ring is $2 \mathrm{~mm}$ from stack

\section{B. 100 HP Motor}

The motor simulation was run over the whole speed range from start to synchronous speed. Fig. 9 shows the difference in torque to be quite small. Without skin effect in the end-ring, the starting torque is $0.6189 \mathrm{kNm}$ compared to $0.6277 \mathrm{kNm}$ which is only a $1.4 \%$ increase. The starting current and input power show similar increases from $601.5 \mathrm{~A}$ to $602.9 \mathrm{~A}$ and $138.5 \mathrm{~kW}$ to $140.2 \mathrm{~kW}$. Fig. 10 shows the variation of the endring resistance and end-ring reactance. These values are referred to the stator using the square of the stator-to-end-ring turns ratio, which is 12034 in this instance. The small ripple is due to the change in the number of radial segments which starts at 5 (preset minimum) and increases to 15 . 
TABLE I

MOTORPARAMETERS

\begin{tabular}{|c|c|c|}
\hline Parameter & $100 \mathrm{hp}$ motor & $300 \mathrm{hp}$ motor \\
\hline Pole Number & 4 & 12 \\
\hline Frequency & $60 \mathrm{~Hz}$ & $60 \mathrm{~Hz}$ \\
\hline Rated Power & $74.6 \mathrm{~kW}$ & $228 \mathrm{~kW}$ \\
\hline RatedSlip & $1.21 \%$ & $1.52 \%$ \\
\hline Rated Voltage & 460 & $4 \mathrm{kV}$ \\
\hline Rated Current & $70 \mathrm{~A}$ & $45 \mathrm{~A}$ \\
\hline Rated Efficiency & $93.7 \%$ & $93.2 \%$ \\
\hline Stator slot Number & 60 & 108 \\
\hline Bar Number & 50 & 148 \\
\hline BarDepth & $31.75 \mathrm{~mm}$ & $25.1 \mathrm{~mm}$ \\
\hline Stack Length & $267 \mathrm{~mm}$ & $270 \mathrm{~mm}$ \\
\hline Rotor Bar Cross-Section Area & $112 \mathrm{~m} \mathrm{~m}^{2}$ & $89.5 \mathrm{~mm}^{2}$ \\
\hline Rotor D iameter & $314.3 \mathrm{~mm}$ & $664 \mathrm{~mm}$ \\
\hline Rotor M aterial & Aluminum & Copper \\
\hline End-ring $\mathrm{H}$ eight & $63.5 \mathrm{~mm}$ & $54.5 \mathrm{~mm}$ \\
\hline End-ring W idth & $9.53 \mathrm{~mm}$ & $12.7 \mathrm{~mm}$ \\
\hline Shape of Ring & Rectangular & Rectangular \\
\hline Distance from Core Pack & $0 \mathrm{~mm}$ & $0 \mathrm{~mm}$ \\
\hline Referred Rated Rotor Resistance* & $0.088 \mathrm{ohm}$ & $0.710 \mathrm{ohm}$ \\
\hline Referred Rated Rotor Reactance* & $1.176 \mathrm{ohm}$ & $13.18 \mathrm{ohm}$ \\
\hline Referred Stand -Still Rotor Resistance* & $0.3728 \mathrm{ohm}$ & $0.902 \mathrm{ohm}$ \\
\hline Referred Stand-Still Rotor Reactance* & $0.8092 \mathrm{ohm}$ & $13.05 \mathrm{ohm}$ \\
\hline ReferredRated BarResistance & $0.0634 \mathrm{ohm}$ & $0.570 \mathrm{ohm}$ \\
\hline ReferredRatedBarReactance & $0.7516 \mathrm{ohm}$ & $12.03 \mathrm{ohm}$ \\
\hline Referred Stand-Still Bar R esistance & $0.3482 \mathrm{ohm}$ & $0.762 \mathrm{ohm}$ \\
\hline Referred Stand-Still Bar Reactance & $0.5848 \mathrm{ohm}$ & $11.92 \mathrm{ohm}$ \\
\hline Stator to End-ring Current Ratio & 67.38 & 55.04 \\
\hline
\end{tabular}

* Excluding skin effect in end-ring.

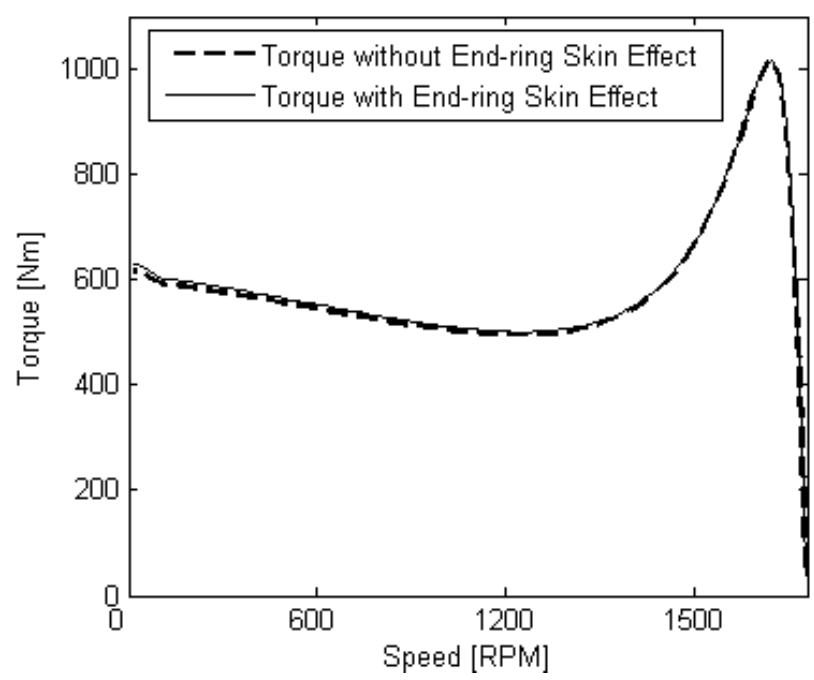

Fig. 9. Torque speed curve for the $100 \mathrm{HP}$ motor

It can be seen that there is little influence on the torque of the skin effect in the end-ring. However, it can be observed that at start, the referred bar resistance is $0.3482 \mathrm{ohm}$ compared to $0.0155 \mathrm{ohm}$ for the end-ring resistance; hence the referred endring resistance is small compared to the referred bar resistance $(4.45 \%)$. The ratio of end-ring-to-bar areas is $605.6 / 112=5.4$ whereas in terms of ratio of end-ring current to bar current this is 4.0. Therefore the end-ring is oversized and can be reduced to increase the starting torque. This will obviously be at the expense of efficiency. However if the width of the ring is halved to $5 \mathrm{~mm}$ the influence of doubling the end-ring resistance is still small with the torque increasing from 0.6347 $\mathrm{kNm}$ to $0.6417 \mathrm{kNm}$ which is only a difference of $1.1 \%$, because the skin effect is decreased by the reduction in ring size.

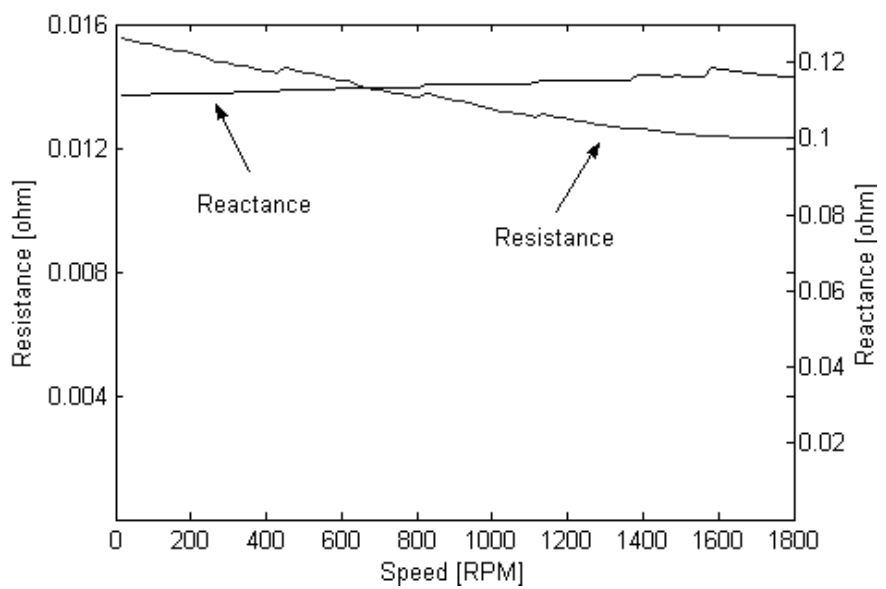

Fig. 10. Variation of end-ring resistance and reactance with speed for $100 \mathrm{HP}$ motor

\section{300 HP Motor}

The 12-pole $300 \mathrm{hp}$ motor simulation was now run producing the torque/speed curve in Fig. 11. The peak torque to starting torque ratio is now much higher. The starting torque is increased from $396.5 \mathrm{Nm}$ to $425.6 \mathrm{Nm}$ which is an increase in starting torque of $7.3 \%$. This illustrates that this work is most relevant to very large machines where the starting torque is quite low. The distribution in the end ring is shown in Fig. 12 at start and Fig. 13 at full load. The markers indicate the centre of the ring segment and the figures represent the full radial width of the ring. At start there are 15 segments with concentration at the edges whereas at full load there are 5 segments since the current variation is more uniform and only the distribution due to the arc of the ring where the resistance path round the inner surface of the ring is less than the outer surface. Whether this distribution would be reflected in reality is open to debate since the current is injected in the top half of the bar and the arc between bars is only 2.43 mechanical degrees. However the ratio in both of these motors of end-ring to bar current is about 4 so that only $25 \%$ of current will be leaving the end-ring into the bar at the ring/bar junction.

The segments in Fig. 12 do seem to be clustered at the edges of the ring. By adjusting $K_{C}$ and $K_{N}$ these can be spread out more if desired.

At standstill the referred bar resistance is $0.762 \mathrm{ohm}$ compared to $0.1889 \mathrm{ohm}$ for the referred end-ring resistance (i.e., $24.8 \%$ ) - obviously showing an increase in the effect of the end-ring. At rated speed the referred bar resistance is 0.570 ohm compared to $0.1263 \mathrm{ohm} \mathrm{(22.1 \% )} \mathrm{Comparing} \mathrm{the} \mathrm{end-}$ ring-to-bar cross section areas: $692.2 / 89.5=7.73$. The endring-to-bar current ratio is 3.94 . Hence, to increase the starting 
torque then the rotor end-rings size can be reduced safely. However when this is done (by reducing the end-ring widths from $12.7 \mathrm{~mm}$ to $8 \mathrm{~mm}$ in the simulations), the starting torque increases from $425.6 \mathrm{Nm}$ to $437.0 \mathrm{Nm}$. However the increase due to skin effect of the end-rings drops from $7.3 \%$ to $5.7 \%$ (the starting torque without end-ring skin effect is $413.6 \mathrm{Nm}$ ).

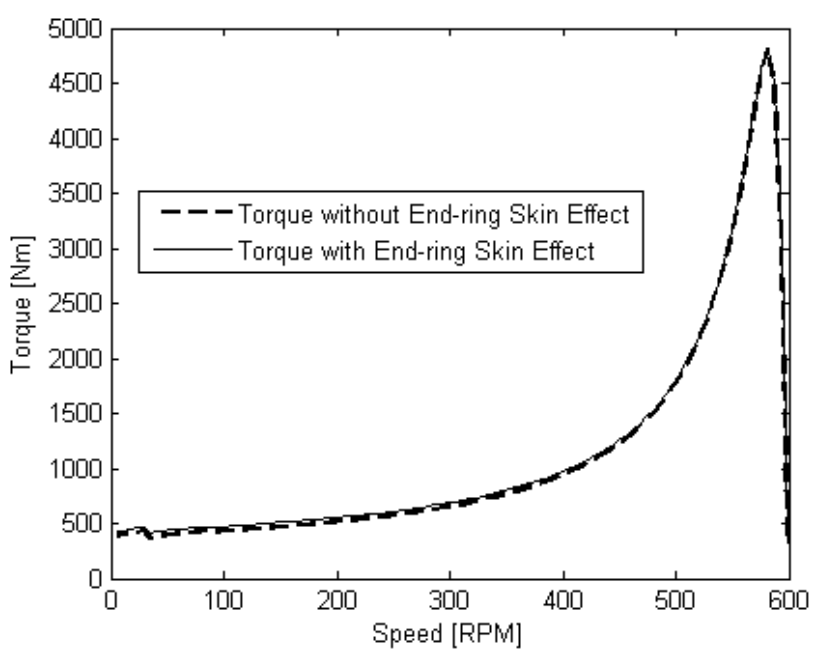

Fig. 11. Torque speed curve for the $300 \mathrm{HP}$ motor

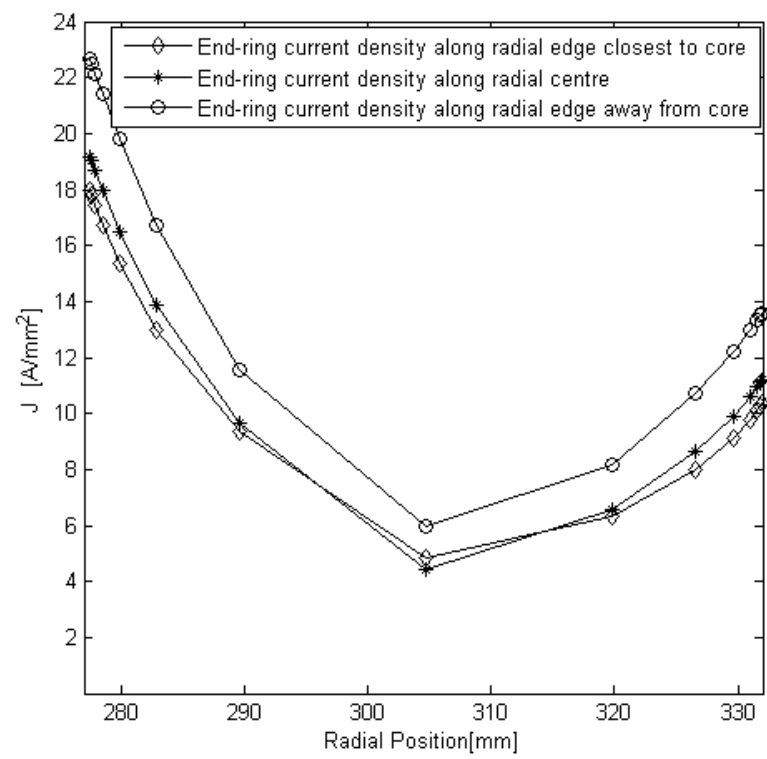

Fig. 12. Current density in the end-ring of the $300 \mathrm{HP}$ motor at start

\section{CONCLUSION}

This paper has highlighted a simple yet effective method for calculating the adjustment necessary to the end-ring impedance in a cage induction motor. This method can be used in conjunction with an analytical circuit method or a $2 \mathrm{~d}$ finite element calculation. It uses a method first developed by Williamson and Mueller [3] but which is developed further to allow for grading of the end-ring elements to cut down on the number of elements and concentrate them at the edges where the change of current from element to element is greatest. The method was verified using a finite element analysis. The first part discussed the modeling method in sufficient detail to allow implementation while the second part illustrated the effect this calculation can have on induction motor performance. The effect on performance is very much a function of the motor design. In this instance a $100 \mathrm{hp}$ motor only showed an increase of $1.4 \%$ in starting torque when skin effect was included in the model whereas for the $300 \mathrm{hp}$ motor (which had a very peaky torque characteristic with low starting torque), the starting torque increased by $7.3 \%$ when end-ring skin effect was included.

If the end-ring skin-effect model is included in the harmonic torque, where the harmonic rotor frequencies are much higher, then the change in end-ring impedance will be even more substantial. In the two motors examined here the asynchronous torques were found to be low.

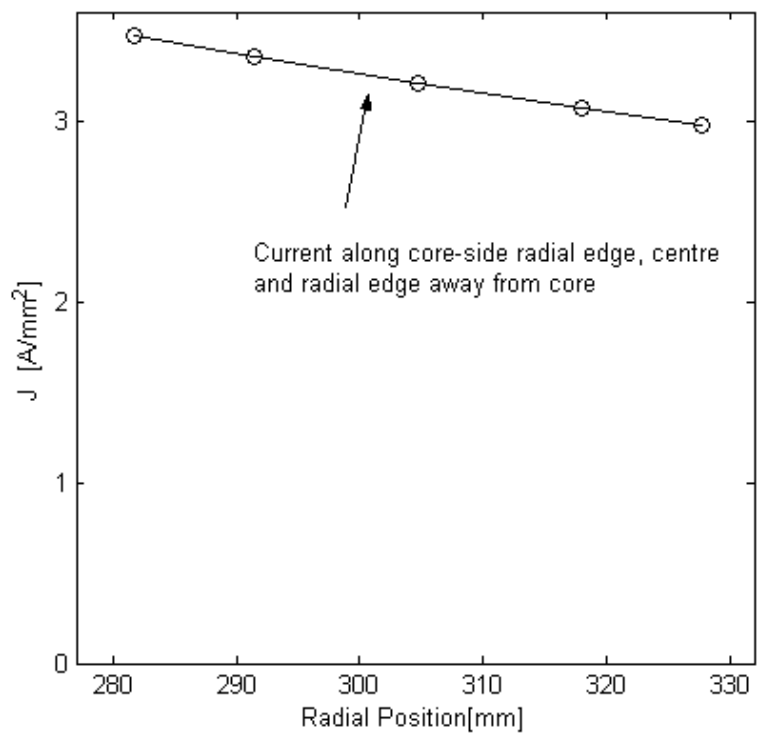

Fig. 13. Current density in the end-ring of the $300 \mathrm{HP}$ motor at full load (slip $=1.5 \%)$

\section{ACKNOWLEDGMENT}

Much of this work was done while the author was Electric Machine Design Manager, SPEED Laboratory, and he is grateful for the support of this work during this time.

\section{REFERENCES}

[1] D. G. Dorrell, TJE Miller and C. Rasmussan, "Inter-bar Current in Induction Machines," in IEEE Trans on Industry Applications, vol. 39, no 3, May/June 2003, pp. 677-684.

[2] P. L. Alger, Induction Machines - Their Behavior and Uses. Gordon and Breach Publishers, Third Edition, 1995, ISBN 2-88449-199-6.

[3] S. Williamson and M. A. Mueller, "Calculation of the impedance of rotor cage end rings," in IEE Proceedings-B, vol. 140, no 1, Jan 1993, pp. 51-60.

[4] R. L. Stoll, The Analysis of Eddy Currents. Oxford University Press, UK, 1974.

[5] K. Yamazaki, "Modification of 2D Nonlinear Time-Stepping Analysis by Limited 3D Analysis for Induction Machines," in IEEE Trans on Magnetics, vol. 33, no 2, March 1997, pp. 1694-1697.

[6] D-W Kim, H-K Jung, S-Y Hahn and C-G Lee, "Three-Dimensional Resistance Calculation in End Ring of Induction Motor by Finite Element Method," in IEEE Trans on Magnetics, vol. 36, no 6, Nov 2000, pp. 3932-3938. 
[7] C. C. Chan, L. Yan, P Chen, Z. Wang and K. T. Chau, "Analysis of Electromagnetic and Thermal fields for Induction Motors During Starting," in IEEE Trans on Energy Conversion, vol. 9, no 1, March 1994, pp. 53-60.

[8] H. Kometani, S. Sakabe and K. Nakanishi, "3-D Electro-Magnetic Analysis of a Cage Induction Motor with Rotor Skew," in IEEE Trans on Energy Conversion, vol. 11, no 2, June 1996, pp. 331-337.

[9] S. Ramo, J. R. Whinnery. And Van Duzer, Fields and Waves in Communications Electronics. J. Whiley and Sons, 1984.

[10] A. C. Smith, "Influence of end-ring shape on induction motor performance," in IEE Conference on Electrical machines and Drives, Cambridge, UK, Sept 1997, pp. 143-147.

David G. Dorrell (M'95) was born in St. Helens, England, in 1965. He obtained the BEng degree from The University of Leeds, UK, in 1988 and the MSc degree from The University in Bradford, UK, in 1989. In 1993 he was awarded a PhD degree by The University of Cambridge, UK, on work related to the calculation of UMP in induction motors.

$\mathrm{He}$ has held lecturing positions with the Robert Gordon University, Scotland and also with The University of Reading, England. He currently holds the post of Senior Lecturer with The University of Glasgow, Scotland. $\mathrm{He}$ has worked for Newage International Ltd, Lincolnshire, England as a Research and Design Engineer and was also employed in the SPEED Laboratory, The University of Glasgow, as Machine Design Engineer for a two year period before being appointed as senior lecturer.

Dr Dorrell has published many papers on electrical machine analysis and design. In 1996 he was awarded The Crompton Premium from the IEE. He is a Chartered Engineer in the UK and a member of the IEE, London. 